\title{
Hepatitis B Virus Screening Patterns amongst Physicians in Hawaii: Changes in a Decade
}

\author{
Chuong T. Tran ${ }^{1,2 *}$, Krista K. Kiyosaki², Linda L. Wong2,3 \\ ${ }^{1}$ California Pacific Medical Center, San Francisco, CA, USA \\ ${ }^{2}$ Department of Surgery, John A. Burns School of Medicine, University of Hawaii, Honolulu, HI, USA \\ ${ }^{3}$ University of Hawaii Cancer Center, Honolulu, HI, USA \\ Email: "huongr@gmail.com, kristakiyosaki@gmail.com, hepatoma@aol.com
}

Received 10 September 2015; accepted 12 October 2015; published 15 October 2015

Copyright (C) 2015 by authors and Scientific Research Publishing Inc.

This work is licensed under the Creative Commons Attribution International License (CC BY). http://creativecommons.org/licenses/by/4.0/

(c) (i) Open Access

\section{Abstract}

Purpose: Hawaii had the highest incidence of liver cancer in the US and had a unique patient population with many immigrants from the Pacific and Asia where Hepatitis B virus (HBV) was endemic. HBV screening in high risk populations was a recommended measure of preventative medicine, thus we sought to examine physician screening patterns for HBV in Hawaii. Methods: We retrospectively analyzed billing claims from 1999 to 2009 from the largest healthcare coverage provider in the state of Hawaii. We identified all patients ( $>18$ years) who underwent HBV screening based on Current Procedural Terminology (CPT) billing codes. We collected data on age, gender and specialty of physicians ordering the screening tests. Analysis was done in 2013 and 2014. Results: Of an estimated 700,000 covered lives, 125,576 patients underwent HBV screening. We stratified the patients into two eras from 1999-2004 $(\mathrm{N}=52,245)$ and 2005-2009 $(\mathrm{N}=73,331)$ to examine temporal trends. In the first era, 30,975 women $(59.3 \%)$ underwent HBV screening, compared to 49,950 women $(69.1 \%)$ screened in the $2005-2009$ era. There absolute number of tests increased, but the proportion done by primary care MDs decreased from $55.6 \%$ to $44.9 \%$. OB/GYN screened $15.6 \%$ in the early era and $26.9 \%$ in later era. Conclusions: There was an increase in women aged 18 - 40 years screened in the 2005-2009 era compared to 1999-2004, most likely due to OB/GYN physicians' screening of prenatal women. Physician education on HBV vaccination/treatment or appropriate referral should include $\mathrm{OB} / \mathrm{GYN}$ as well as primary care physicians.

\section{Keywords}

CPT Codes, Hepatitis B, HBV Screening Patterns, Hawaii

\footnotetext{
*Corresponding author.
}

How to cite this paper: Tran, C.T., Kiyosaki, K.K. and Wong, L.L. (2015) Hepatitis B Virus Screening Patterns amongst Physicians in Hawaii: Changes in a Decade. Open Journal of Gastroenterology, 5, 139-145. 


\section{Introduction}

Of the estimated 800,000, 1.4 million people in the U.S. with hepatitis B infection (HBV), 65\% are unaware of their infection [1] [2]. Although US physicians perform routine immunization of HBV at birth, immigrants from endemic areas (including Asia and the Pacific Islands) may be contributing to the persistent presence of HBV. The prevalence of HBV infection in the U.S. is estimated to be $0.4 \%$; however, this likely underestimates the problem as the figure is based on surveys that under-represented groups with a high prevalence of HBV, especially foreign-born minorities [3]. A recent analysis using the Racial and Ethnic Approaches to Community Health survey data (2009-2010) demonstrated that only half of racial/ethnic minority persons receive HBV screening, and only half of those who tested positive eventually received treatment. Furthermore, this study shows a decreased likelihood HBV screening and treatment in those who are female, without health insurance, and without college education [4]. In hopes of addressing these disparities, the U.S. Preventive Services Task Force in 2014 issued a recommendation for HBV screening in those who were born in countries with a high prevalence of HBV, not vaccinated as infants, HIV positive, immunosuppressed or on hemodialysis [5].

Hawaii is the ideal place to study screening and treatment of HBV in the U.S. Census data shows that $38.7 \%$ of the population is Asian, $8.8 \%$ are Pacific Islanders, and $17.2 \%$ are born in a foreign country [6]. The prevalence of HBV in Hawaii is estimated at 3.6\% with especially high prevalence in Vietnamese, Chinese and Pacific Islanders [7]. In addition, there are 8000 migrants from Micronesia/Marshall Islands currently living in Hawaii, as they are able to travel without the need for visas [8]. The incidence of HBV in these small Pacific Island nations is reportedly as high as $15 \%$ [9]. As a consequence, Hawaii has the highest incidence and second highest death rate of hepatocellular cancer in the U.S. and nearly $40 \%$ of this is HBV-related [10] [11]. Due to Hawaii's unique demographics with a growing population deemed as high risk for HBV, we seek to understand physicians' HBV screening practice patterns by querying a large administrative billing database over a 10-year period.

\section{Methods}

We first obtained exemption for this study was from the University of Hawaii Institutional Review Board, as documented in the Code of Federal Regulation at 45 CRF 46. We then submitted our protocol and request for data to the largest provider of health care coverage in the State of Hawaii (about $65 \%$ of insured people in Hawaii). This insurer has a breadth of coverage including a health maintenance organization, affiliation with the Blue Cross Association and a program that contracts with the State of Hawaii for Medicaid services. This study was approved by the insurer's Quality Management department. This provider's data elements and IT departments provided support in generating anonymous patient data for this study.

All patients in the database who underwent HBV screening as defined by CPT billing codes for blood tests (codes: hepatitis serology panel, 80059; HBcAb, 86704; HBcAb IgM, 86705; HBsAb, 86706; HBeAb, 86707; HBsAg, 87340, 87341) between January 1, 1999 and December 31, 2009 were available for inclusion in the study. Patients who were less than 18 years old were excluded from the study. HBV blood tests obtained on patients who were hospitalized or seen in the emergency department were excluded in order to eliminate those patients with an acute liver-related illness and health care workers involved in needle stick injuries or exposures. Due to the insurance provider's regulations, patients with sensitive diagnoses (including mental health issues, diagnosed drug abuse, and HIV positivity) were excluded from analysis. However, this group accounted for $<4 \%$ of the patient population. Each patient had up to 4 diagnosis codes designated, however the listed diagnoses varied widely with frequent listings that included the terms "other", "unspecified" or "not otherwise specified" (for example, "ascites, unspecified" may have been related to liver disease, renal disease or heart failure). The diagnosis data was not reliable enough to determine if the HBV tests were done for screening or another diagnosis and were not used in the analysis.

Data collected included patient age and gender and ordering physician's specialty and location by island. Tests done on the same patient in the same year were counted as one test to eliminate the possibility of testing for confirmation purposes. On the other hand, tests done on the same patient in different years across the study period were kept as separate screening tests to recognize that they may have been done for different reasons (new risk factors, multiple pregnancies, etc.). Physician specialties were categorized as primary care (internal medicine, family medicine, pediatrics), OBGYN, gastroenterology, non-GI medical specialties, surgical specialties, and non-physician providers (nurse practitioner, physician assistant, etc.). Physician location was divided 
into Oahu (urban setting) versus all of the other Hawaiian Islands (rural setting). Once we defined our patient population we used the data to examine temporal trends in HBV screening patterns, analyzing patient age, patient gender, and physician specialties. To examine temporal trends, we stratified tests into two eras: 1999-2004 and 2005-2009. We also performed comparisons between gender, age groups (18 - 40 years, 41 - 64 years, 65 years and older), provider specialties and geographic location (urban vs. rural).

Data was initially provided in Excel, version 2010. Numerical values were analyzed with Student's $t$-test with significance set at $p=0.05$. Odds-ratio on gender was compared to the population of Hawaii based on U.S. Census data from 1999 to 2009 [6] [12]. Analyses were performed in 2013 and 2014.

\section{Results}

Of an estimated 700,000 covered lives, there were 100,711 unique patients with 125,576 HBV screening tests from 1999-2009. 44,651 screening tests were performed on men and 80,925 were done on women. The breakdown of tests done by age groups was as followed: 63,470 in the 18 to 40 age group, 53,154 in the 41 to 64 age group, and 8952 in the 65 or greater group (see Table 1). The mean age was 41.9 years. Based on U.S. Census data on Hawaii's population for 2009, we estimate that about 11.6\% of Hawaii's residence was screened for hepatitis B between 1999 and 2009 [6].

Year-to-year analysis revealed a steady increase in the number of screening tests ordered by the primary care group (mean increase of 296 tests per year), with the highest increase between 2008 and 2009 of 1221 tests. From 1999 to 2009, the number of HBV tests ordered by primary care physicians increased by $64 \%$. The OBGYN group experienced a larger rate of increase in the number of screening tests, from 1085 tests in 1999 to 5107 tests in 2009. The most dramatic year-to-year difference occurred between 2006 and 2007, when the number of screening tests increased from 2732 to 4457 tests (a 63\% increase). Another dramatic increase in screening tests ordered was seen in the non-physician providers group, which increased 17.2 fold over the study period, with $39 \mathrm{HBV}$ tests ordered in 1999 and $674 \mathrm{HBV}$ tests in 2009. Yearly changes in the number of tests ordered by the medical and surgical specialties groups were insignificant (see Figure 1).

There were 52,245 tests in the first era (1999-2004) and 73,331 tests in the second era (2005-2009). In the first era, 30,975 women (59.3\%) underwent testing, compared to 49,950 women (68.1\%) in the second era. Using U.S. Census data, the odds ratio for women being screened over the 10 -year period was 1.83 (95\% CI 1.80 $1.85, p=0.001$ ) [6]. There was an overall increase in screening tests performed between the two eras, but the proportion of tests ordered by primary care MDs decreased from $55.6 \%$ to $44.9 \%$. Likewise, the proportion of screening tests done by GI physicians decreased almost by half between the two eras (6.2\% to 3.8\%). OBGYN physicians screened $15.6 \%$ (8142 tests) in the first and $26.9 \%$ (19,722 tests) in the second era. Changes in screening by non-GI specialists and surgical specialists were insignificant between the two eras. Lastly, screening tests done by non-physician providers significantly increased, doubled in proportion $(1.3 \%$ to $2.8 \%)$ and

Table 1. Number of HBV screening tests divided by era (1999-2004 and 2005-2009), gender and age groups. The shift in the number of screening tests to women of younger age groups between the eras is statistically significant, as shown by $p$-values.

\begin{tabular}{|c|c|c|c|}
\hline \multicolumn{4}{|c|}{ HBV screening tests distribution by gender and age groups } \\
\hline & 1999-2004 & 2005-2009 & $P$-value \\
\hline Number of screening tests & 52,245 & 73,331 & \\
\hline Number of tests on women & $30,975(59.3 \%)$ & $49,950(68.1 \%)$ & $<0.0001$ \\
\hline Mean age of women (years) & $40.4 \pm 14.55$ & $38.3 \pm 15.21$ & 0.0001 \\
\hline Mean age of men (years) & $46.1 \pm 13.34$ & $47.8 \pm 14.76$ & 0.0001 \\
\hline Ages $18-40$ & $24,084(46.1 \%)$ & $39,386(53.7 \%)$ & 0.008 \\
\hline Ages $41-64$ & $24,824(47.5 \%)$ & 28,330 (38.6\%) & \\
\hline Ages 65+ & 3337 (6.4\%) & 5615 (7.7\%) & \\
\hline
\end{tabular}




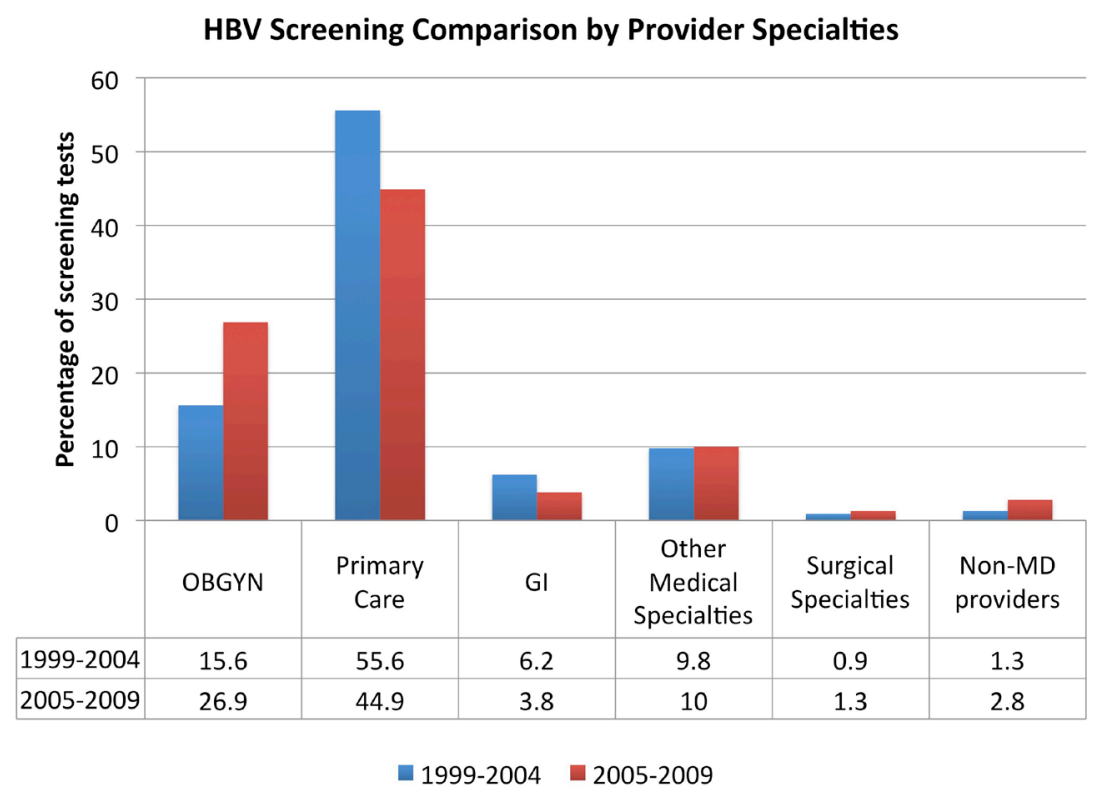

Figure 1. HBV Screening tests by provider specialties, expressed in percent of total number of tests.

tripled in number (679 to 2053) between the two eras (see Figure 2).

In terms of analysis by provider specialties and geographic locations, 13,975 of the 125,576 screening tests (11.1\%) were excluded from the analysis because they were marked as unknown provider specialty $(13,057)$ and/or unknown provider geographic location $(11,975)$. The primary care group on Oahu ordered 4466 more screening tests in the second era than the first era, a 20\% increase. The non-Oahu primary care group had a 5.8\% reduction in tests ordered (393 tests) in the second era. As for the OBGYN group, the number of screening tests for OBGYN providers on Oahu increased from 4728 tests in the first era to 14,408 tests in the second; a 205\% increase. The non-Oahu OBGYN group had a more modest increase of 1892 tests (56\% increase). The nonphysician providers group also had an increase in screening tests ordered, with the more dramatic rise from 321 to 1622 (5-fold increase) tests in the Oahu providers (see Figure 3). There was also an increase in the number screening tests ordered by the surgical specialties group, although this group only makes up about $1 \%$ of the total screening tests.

\section{Discussion}

It is unclear what portion of Hawaii's population needs HBV screening based on the Centers for Disease Control and Prevention (CDC) guidelines. With the high proportion of foreign-born residents who emigrate from highrisk countries, the number of people who need HBV screening in Hawaii is certainly higher than most places in the U.S. Although we cannot evaluate the effectiveness of HBV testing in identifying and appropriately treating HBV, this study has shown that an estimated $11.6 \%$ of Hawaii's population has had HBV testing. The increase in HBV testing over time is also encouraging in the goal of adequately screening Hawaii's population.

Primary care physicians are proved to be the main group performing HBV screening in Hawaii, accounting for about half of the number of screening tests ordered during the study period. While these statistics are not surprising, they emphasize the importance of primary care physicians in HBV prevention. Several studies have been done in attempt to understand HBV screening practices of physicians in the U.S. Ferrante et al. [13] sent out surveys to 1000 family medicine physicians in New Jersey regarding screening and counseling for HBV. Of the 217 respondents, 32\% of these physicians score less than $67 \%$ in answering questions about screening guidelines for HBV [13]. Another study by Foster et al. in 2011 [14] surveyed physicians in the disciplines of internal medicine, OBGYN, family medicine, and gastroenterology across the continental U.S. regarding screening of HBV. Of 1000 physicians who receive the survey, 151 respond and $60 \%$ of these physicians correctly recognize the CDC screening guidelines for $\mathrm{HBV}$, while $40 \%$ are unaware that heterosexual inter- 


\section{Increase in HBV screening tests over 10 years using 1999 as the index year}

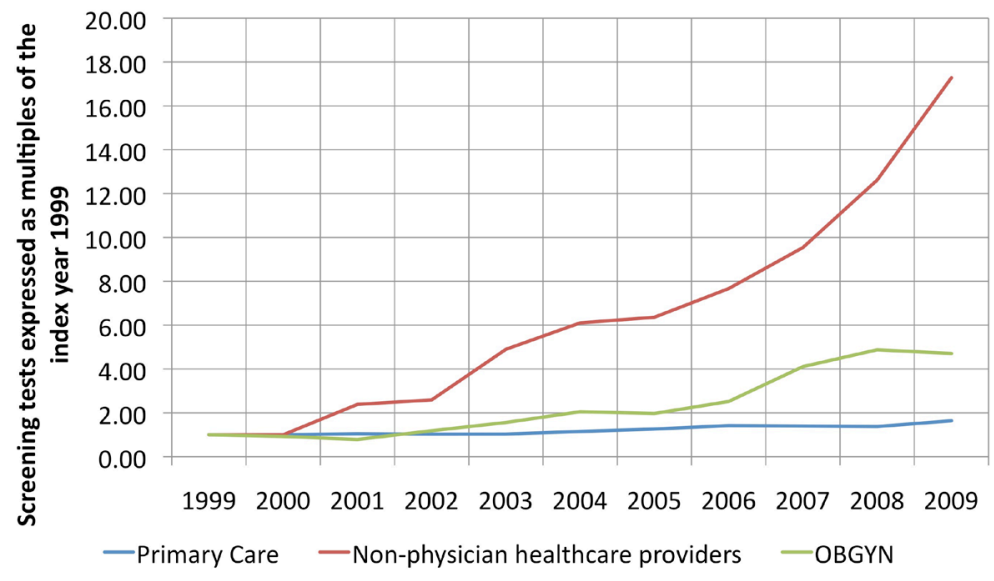

Figure 2. This is the increase in ordering of screening tests over 10 years by specialty with the index year of 1999. The largest increase is in non-physician providers, which increased 17-fold from 1999.

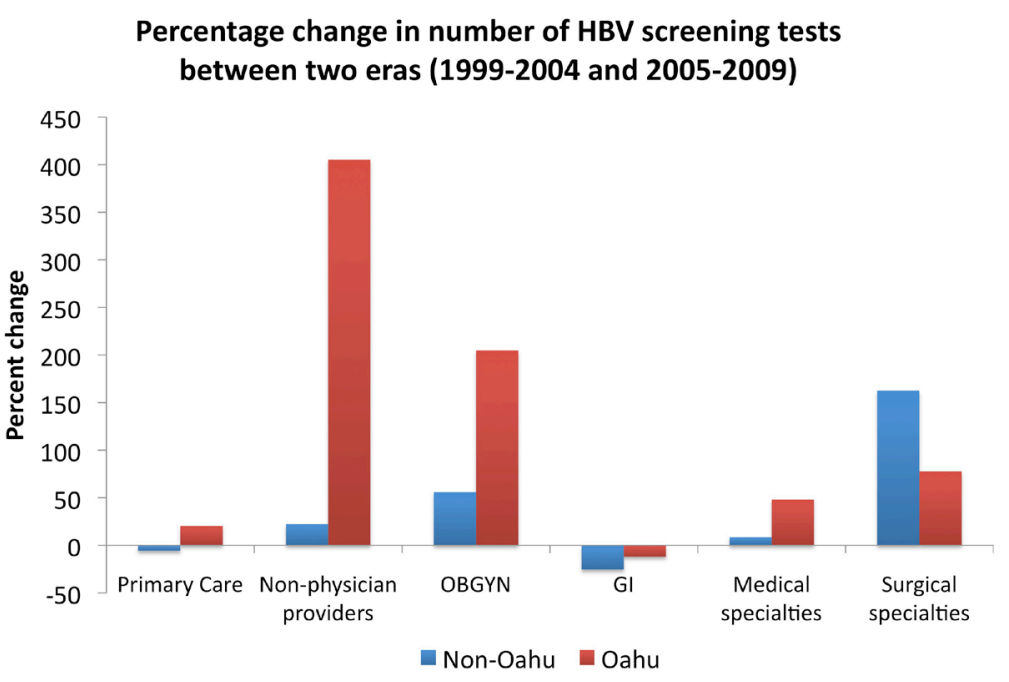

Figure 3. Percentage increase or reduction in the number of HBV screening tests between two eras: 1999-2004 and 2005-2009. On Oahu, screening tests ordered by non-physician providers increased by $405 \%$, and tests ordered by OBGYN increased by $205 \%$.

course is the most common route of HBV transmission in the U.S. [14]. These studies reveal the need for improvement in awareness and application of HBV screening guidelines amongst physicians.

While primary care physicians continue to assume the dominant role in HBV screening, OBGYN physicians contribute significantly to screening efforts, particularly in the latter era of the study. The number of HBV screening tests ordered by OBGYN physicians more than doubled between the two eras, with the most dramatic increase in young women of childbearing age (age group 18 - 40 years). The mean age of women being screened is reduced from 40.4 years to 38.3 years $(p=0.0001)$ between the two eras, while the mean age of men being screened increases from 46.1 years to 47.8 years $(p=0.0001)$. In fact, when only the primary care group is analyzed, females are significantly more likely to be screened for HBV in the later era $(p=0.001)$. The most significant increase in screening tests ordered is observed in the Oahu OBGYN group, with a 3-fold increase between the first and second era of the study.

It is not clear why OBGYN physicians have such marked increase in HBV screening, but perhaps changes in 
birthrate or maternal ethnicity might have been contributed. Data from the Hawaii Health Information Corporation showed that the birth rates were relatively stable on Oahu at 14.63 per 1000 population (1999-2004) compared to 14.88 per 1000 (2005-2009) and slightly increased in the non-Oahu population 13.54 per 1000 in the first era (1999-2004) versus 14.31 per 1000 in the second era (2005-2009). The absolute number of births in the first era is 105,306 and in the second era is 94,155 [12]. Therefore it is unlikely that changes in number of births are a major contributing factor to the increase in screening tests.

Perhaps OBGYN physicians have become increasingly aware of HBV screening recommendations with time. In 2005, the CDC published a revision to the prevention and immunization strategies for HBV, recommending that all pregnant women be screened for HBV in the first trimester [15]. This revision may have increased HBV screening however the same recommendation was initially made by the American College of Obstetricians and Gynecologists (ACOG) and the CDC over 20 years ago [16] [17]. Perhaps better recognition of these ACOG and CDC recommendations contributing to the increased awareness and application of HBV screening guidelines. Regardless of reasons, HBV screening practices by OBGYN physicians make this group a sometimes unrecognized, but very important part of the surveillance effort.

In addition to HBV screening, appropriate referral or relay of positive results play an equally important role in patient care. A survey of 593 OBGYN physicians in 2003 showed that $89 \%$ routinely screened for HBV in their prenatal patients; only half of these physicians routinely reported positive results to the health department, and approximately $10 \%$ did not routinely report positive results to their patients' labor and delivery hospitals [18]. Past surveys also revealed that some OBGYN physicians believed screening and vaccination were not the responsibility of the obstetrician [18] [19]. Furthermore, another study surveyed 732 pediatricians in California and found that only $12.9 \%$ of surveyed pediatricians reported that they always received maternal HBV serology results from obstetrical nurses, and only $4.2 \%$ always received a written letter about maternal HBV serology results from the obstetrician [20]. Efforts are needed to not only increase OBGYN physician awareness about HBV screening but to help them understand the need for appropriate vaccination and linkage to care.

Finally, HBV screening education should also target non-physician or mid-level providers. The 17 -fold increase in HBV screening tests ordered by non-physician providers from the beginning to the end of our study period, most likely reflects the rising number of these providers in Hawaii, particularly on urban Oahu (see Figure 2). Since the 1990's, more training programs for nurse practitioners and physician assistants have been established, along with legal expansion in the scope of practice of these providers [21]. The growth of non-physician providers is expected to continue at accelerating rates in virtually all medical specialties [22] [23].

This study was limited as it was based on an administrative billing database and it was only as accurate as the office/laboratory staff members who have entered the various billing codes. The database had incomplete information on patient demographics and we were unable to determine the patients' ethnicity, birthplace or specific risk factors that would warrant HBV screening. Specific diagnoses were not consistent enough to help determine the reason for HBV testing. Finally, the billing codes were only able to indicate that a test was performed so we could not determine if the test was positive or if there was a subsequent linkage to care.

In spite of these limitations, this is a very large sample size that represents at least $65 \%$ of Hawaii's population over a 10-year period. Although we cannot determine exact HBV screening practices, we can see trends in ordering of HBV testing among various groups of physicians over time. Clearly OBGYN physicians and nonphysician mid-level health care providers are playing an increasingly important role in HBV screening. Efforts to educate and increase awareness of screening guidelines as well as appropriate treatment/referral should be targeted to these groups of providers.

\section{Acknowledgements}

This study was made possible through the support and help of the HMSA Data Elements and IT teams.

\section{Conflict of Interest}

We have no conflict of interest to report.

\section{References}

[1] Lin, S.Y., Chang, E.T. and So, S.K. (2007) Why We Should Routinely Screen Asian American Adults for Hepatitis B: A Cross Sectional Study of Asians in California. Hepatology, 46, 1034-1040. http://dx.doi.org/10.1002/hep.21784 
[2] Hagan, H., Campbell, J., Thiede, H., Strathdee, S., Oueller, I., Kapadia, F., et al. (2006) Self Reported Hepatitis C Virus Antibody Status and Risk Behavior in Young Injectors. Public Health Reports, 121, 710-719.

[3] Kim, W.R. (2009) Epidemiology of Hepatitis B in the United States. Hepatology, 49, 28-34. http://dx.doi.org/10.1002/hep.22975

[4] Hu, D.J., Xing, J., Tohme, R.A., Liao, Y., Pollack, H., Ward, J.W., et al. (2013) Hepatitis B Testing and Access to Care among Racial and Ethnic Minorities in Selected Communities across the United States. Hepatology, 58, 856-862. http://dx.doi.org/10.1002/hep.26286

[5] (2014) U.S. Preventive Services Task Force Recommendations on Hepatitis B Screening. http://www.uspreventiveservicestaskforce.org/bulletins/hepbbulletin.pdf

[6] U.S. Census Bureau, Fact sheet for Hawaii. www.census.gov

[7] Tsai, N., Holck, P.S., Wong, L.L. and Ricalde, A.A. (2008) Seroepidemiology of Hepatitis B Virus Infection: Analysis of Mass Screening in Hawaii. Hepatology International, 2, 478-485. http://dx.doi.org/10.1007/s12072-008-9103-0

[8] Pobutsky, AM., Buenconsejo-lum, L., Chow, C., Palafox, N. and Maskarinec, G.G. (2005) Micronesian Migrants in Hawaii: Health Issues and Culturally Appropriate, Community Based Solutions. Californian Journal of Health Promotion, 3, 59-72.

[9] Kowdley, K., Wang, C.C., Roberts, H. and Brosqart, C.L. (2012) Prevalence of Chronic Hepatitis B among ForeignBorn Persons Living in the United States by Country of Orgin. Hepatology, 56, 422-433. http://dx.doi.org/10.1002/hep.24804

[10] National Cancer Institute. SEER data. www.cancer.gov/statistics

[11] Wong, L.L., Hernandez, B., Kwee, S., Albright, C., Okimoto, G. and Tsai, N. (2012) Healthcare Disparities in Asians and Pacific Islanders with Hepatocellular Cancer. American Journal of Surgery, 203, 726-732. http://dx.doi.org/10.1016/j.amjsurg.2011.06.055

[12] Health Trends in Hawaii. www.healthtrends.org

[13] Ferrante, J.M., Winston, D.G., Chen, P.H. and de la Torre, A.N. (2008) Family Physicians’ Knowledge and Screening of Chronic Hepatitis and Liver Cancer. Family Medicine, 40, 345-351.

[14] Foster, T., Hon, H., Kanwal, F., Han, S. and Spiegel, B. (2011) Screening High Risk Individuals for Hepatitis B: Physician Knowledge, Attitudes, and Beliefs. Digestive Diseases and Sciences, Published Online.

[15] CDC (2005) A Comprehensive Immunization Strategy to Eliminate Transmission of Hepatitis B Virus Infection in the United States: Recommendations of the Advisory Committee on Immunization Practices (ACIP). Part 1: Immunization of Infants, Children, and Adolescents. MMWR, 54, 1-23.

[16] CDC (1990) Protection against Viral Hepatitis: Recommendation of the Immunization Practices Advisory Committee (ACIP). MMWR, 39, 1-26.

[17] American Academy of Pediatrics, American College of Obstetricians and Gynecologists (1992) Guidelines for Perinatal Care. 3rd Edition, American Academy of Pediatrics, Elk Grove Village.

[18] Schrag, S.J., Fiore, A.E., Gonik, B., Malik, T., Reef, S., Singleton, J.A., et al. (2003) Vaccination and Perinatal Infection Prevention Practices among Obstetrician-Gynecologists. Obstetrics \& Gynecology, 101, 704-710. http://dx.doi.org/10.1016/S0029-7844(03)00010-3

[19] Gonik, B., Jones, T., Contreras, D., Fasano, N. and Roberts, C. (2000) The Obstetrician-Gynecologist’s Role in Vaccine-Preventable Disease and Immunization. Obstetrics \& Gynecology, 96, 81-84. http://dx.doi.org/10.1016/S0029-7844(00)00860-7

[20] Rosenthal, P., Wood, D.L., Greenspoon, J.S. and Pereyra, M. (1995) Hepatitis B Virus Serology in Pregnant Women: Transmittal of Results from Obstetricians to Pediatricians in California. The Pediatric Infectious Disease Journal, 14, 927-931. http://dx.doi.org/10.1097/00006454-199511000-00001

[21] Druss, B.G., Marcus, S.C., Olfson, M., Tanielian, T. and Pincus, H.A. (2003) Trends in Care by Nonphysician Clinicians in the United States. New England Journal of Medicine, 348, 130-137. http://dx.doi.org/10.1056/NEJMsa020993

[22] Moses, R.E. and Feld, A.D. (2007) Physician Liability for Medical Errors of Nonphysician Clinicians: Nurse Practitioners and Physician Assistants. American Journal of Gastroenterology, 102, 6-9. http://dx.doi.org/10.1111/j.1572-0241.2006.00804.x

[23] Nestor, M.S. (2005) The Use of Mid-Level Providers in Dermatology: A Liability Risk? Seminars in Cutaneous Medicine \& Surgery, 24, 148-151. http://dx.doi.org/10.1016/j.sder.2005.06.003 